\title{
CAPÍTULO VIII \\ Religar saberes y sentires para reinventar la convivencialidad
}

\author{
Rubén Fontalvo Peralta ${ }^{1}$
}

1 Sociólogo, Magíster en Educación y Filosofía Latinoamericana. Candidato a Doctor en Ciencias de la Educación. Miembro del Grupo de Investigación Educación Ciencias Sociales y Humanas. Universidad Simón Bolívar.

rfontalvo11@unisimonbolivar.edu.co 


\title{
RESUMEN
}

El presente artículo de investigación es una indagación crítica de la necesidad de comprender la relación compleja entre el sentir y el pensar; de manera propositiva plantea la necesidad de reinventar el pensar para dar cuenta del conjunto del ser integral, tanto en la micropolítica como en la macropolítica, en una dinámica de reinvención de la convivencialidad humana y armonía con la naturaleza. Propone una transición ecológica y cultural profunda, que se oriente a sistemas de organización socio-naturales muy diferentes a los actuales, que trasciendan los modelos del sistema capitalista. Esta trama conduce el pensamiento y las acciones humanas a la necesidad de religar los múltiples saberes, en un diálogo creativo entre lo que se investiga y la formación profesional universitaria.

Palabras clave: Antropoceno, buen vivir, cosmodernidad, decolonialidad, micropolítica, sentipensar.

\begin{abstract}
Thisresearchis a criticindagationaboutneeding of understandthecomplexrelationshipbetweenfeeling and thinking, in a propositiveway poses theneeding of reinventthinking to giveanaccount of integral beingboth in micropolitics and macropolitics in a reinventiondinamic of human conviviality and harmonywithenvironment. Itproposesanecologic and cultural deeptransition, which leads to socionaturalorganizationsystemsthat are diferent to current andthattrascendmodels of capitalistsystem. Thisissueguidesthinking and human acts to theneeding of relinkmultipleknowledges, in a creative dialogue betweenwhatisresearching and theuniversityeducation.
\end{abstract}

Key words: Anthropocene, Good living, Cosmodernity, Decoloniality, micropolitics, thinking-feeling. 


\section{Introducción}

La humanidad se enfrenta a múltiples problemas que exigen una respuesta global, en la que la ciencia es un componente esencial y decisivo a la hora de abordar los retos apremiantes si se aprovecha todo su potencial combinado con los conocimientos de las comunidades locales. Se enfrentan problemas históricos aún no resueltos y nuevos problemas que requieren nuevas formas de pensar las soluciones que beneficien a toda la humanidad reinventando la convivencialidad, comprendida esta como la cordialidad entre las personas individual y colectivamente, el compartir las alegrías, las amistades, la comunicación sincera, los placeres y los displaceres. Es la relación compleja entre el sentir y el pensar, en la que el pensar requiere dar cuenta del conjunto del ser integral, tanto en la micropolítica como en la macropolítica.

Esta trama conduce el pensamiento y las acciones humanas a la necesidad de religar los múltiples saberes y sentires, en un diálogo creativo entre lo que investigamos y hacemos en la academia, sector empresarial, gobierno y los conocimientos que los movimientos sociales (indígenas, afrodescendientes, ambientalistas, campesinos, mujeres, nuevas ciudadanías) generan, aplican y resisten por fuera de la academia. Replantear esta lógica de crear conocimientos es quizás uno de los esfuerzos con los que se enfrenta el profesional del Trabajo Social.

Por fuerza de la crisis múltiple que experimenta el sistema antropoceno, caracterizado por el papel central que desempeñamos los seres humanos como fuerza geológica determinante, la comprensión de las causas, consecuencias y respuestas que se han de dar para afrontarla, deben ser de índole social.

Hoy podemos considerar que estamos en una dinámica en transición epistemológica si consideramos que los paradigmas dominantes ya han dado cuenta de sus limitaciones, lo que es una buena oportunidad para reinventar y activar la creatividad para cuidar e innovar. La crisis ecológica y social ha llevado a muchos investigadores a proponer una transición ecológica y cultural profun- 
da, orientada a sistemas de organización socio-naturales muy diferentes a los actuales que trascienden los modelos del sistema de dominación.

Los discursos en transición son caracterizados como emergentes, haciendo de la emergencia una categoría epistemológica indispensable para comprender y actuar en el mundo de hoy, lo que quiere decir que, hay que tener presente la lucidez del pensar y el sentir, e incluir un saber ético distinto de una conciencia moral, para dar cuenta del inconsciente colonial capitalístico que comanda al sujeto moderno que todavía encarnamos (Guattari, 2005). La fuerza del pensamiento y las utopías estarán orientadas a una verdadera transición de civilización.

El siglo XXI le lanzó nuevos desafíos a la misión de la universidad, relacionados con los aportes que la investigación científica puede hacer para la toma de decisiones, en una etapa caracterizada como de transición en relación con un horizonte de desarrollo sostenible e inclusivo que evolucione al Buen Vivir. Esto implica para la educación superior que la formación profesional y las investigaciones tengan aplicabilidad en sus respectivos contextos, complementándose con la investigación fundamental o básica que sin tener en mente su aplicación específica inmediata, lleva a nuevos conocimientos que así como conduce a la creación de nuevas tecnologías, también ofrece nuevos enfoques en el saber y en el emocionar para el saber hacer.

La ciencia fundamental o básica y la ciencia aplicada son interdependientes, se complementan para ofrecer soluciones innovadoras frente a las incertidumbres, debido a las complejidades y el alcance de los problemas que aumentan de manera acelerada. En este escenario, las investigaciones sociales constituyen una exigencia para dar cuenta de manera transformadora de problemas, como las crecientes desigualdades sociales.

\section{La condición humana en el contexto del antropoceno}

Estamos viviendo una expresión máxima de la incomprensión humana, pues 
tenemos abundante información, saberes, conocimiento, datos, estrategias que nos permiten identificar las causas, interés, afanes que han generado para la humanidad vivir los últimos 70 años en medio de guerras, grandes inversiones en armas, muertes, destrucciones, con todo lo que esto ha generado en la convivencia humana y el medioambiente con la amenaza del cambio climático. Sin embargo, seguimos sometidos y confiando en los mismos caminos del pensamiento y las acciones que nos inducen e imponen un paradigma que cada vez más muestra sus insuficiencias, pero que se resiste a dejar de existir, razón por la cual se requiere activar y reinventar las compresiones, las creatividades, las solidaridades, la convivencialidad.

La incomprensión al lado de las arrogancias y codicias es compañera de la estupidez que es seguir un camino, una información, un dogma, un paradigma a sabiendas de las consecuencias destructoras que generan las acciones que emprendemos por la incomprensión de sus implicaciones. Pero esta incomprensión es inducida por un sistema que se implementa por todos los procesos, siendo uno de ellos la educación, que enseña a aprender incapacidades así como estimular deseos que actúan en la subjetividad a partir de la experiencia inmediata, para seguir sometidos y en estado de ignorancia, dando origen a una disciplina como la Agnotología, que es el arte de reducir las mentes, con el engaño y la producción deliberada de la ignorancia, con el propósito de bloquear o retrasar la adopción de ideas.

Cada vez más se reconoce que los problemas ecológicos, sus riesgos, crisis, desafíos, conflictos, no se dan aisladamente y no ocurren en sistemas distintos y autónomos, aislados en el medioambiente natural por un lado y el sistema social por otro lado, sino que forman parte de un sistema complejo de relaciones interdependientes que podemos denominar antropo-bio-cósmico. Las medidas tecno-económicas y sus implicaciones en los cambios ambientales globales como el cambio climático, que constituye hoy uno de los problemas más trascendentales para la convivencialidad de la humanidad y la vida de todas las especies vivientes, tienen una dimensión planetaria, por eso se necesitan acciones sociales y políticas planetarias. 
Estamos viviendo una época en la que se han producido alteraciones sustanciales en nuestro planeta, que ponen en peligro la aniquilación de toda la especie humana: la elevación del nivel del mar por deshielizacion puede anticipar que dentro de algunos siglos puede desaparecer todo el hielo del planeta elevando el nivel del mar; desde ese punto de vista no es la tecnología la que salvará nuestra especie, más sí un cambio radical de nuestra visión de la realidad, considerando simultáneamente las interacciones y complementariedades de todos los niveles de la realidad por un futuro sustentable.

Los científicos saben hoy con mucha certeza que esos cambios están motivados principalmente por las actividades de la especie humana, que al modificar la composición de la atmosfera de la Tierra, se llega al riesgo de destruir la propia civilización y la especie. El cambio ambiental global es obra de los seres humanos, que al transformar los entornos globales configuran, individual y colectivamente, el rumbo de la evolución de la sociedad; en armonía con la naturaleza propone la necesidad de convertirnos en los copilotos de un planeta caliente en medio de un cosmos en expansión.

Frente a esta circunstancia se está vislumbrando una nueva era: la cosmodernidad, fundamentada en una nueva visión de las interacciones ciencia, educación, sociedad, espiritualidad. La cosmodernidad significa que toda entidad en el universo es definida por su relación con otras entidades. Se retoma así la antigua idea de cosmos donde todos somos partícipes activos.

Las ciencias en general y la educación en todos los niveles, tienen que desempeñar un papel fundamental para lograr que la sociedad comprenda mejor qué significa vivir y, hacer evolucionar el pensar y sentir de lo humano en el antropoceno, para tener conciencia de las vulnerabilidades y desigualdades así como de las posibilidades, las responsabilidades y la obligación de actuar para la construcción de un futuro sustentable que evolucione al Buen Vivir. Complementario a esto, se requiere una inspiración en el contexto del sentipensar popularizado por Fals Borda, desde las concepciones enraizadas culturalmente en las comunidades de la Costa Atlántica colombiana. 


\section{La sociedad es una inacabable secuencia de ambigüedades y bifurcaciones}

Nuestra vida social es indudablemente la búsqueda y elección de una posibilidad dentro de infinidad de posibilidades, siendo el resultado de sus propias bifurcaciones, y la civilización occidental, ha optado por unas vías y ocultado, negado, excluido, reprimido o dejado a un lado otras vías (Fontalvo, 2009).

$\mathrm{Al}$ hacer un análisis histórico de las ambivalencias de la sociedad Max Neef (2011) identifica cómo a principios del siglo XIII, en Italia un joven llamado Giovanni Di Bernardone, siendo aún muy joven y muy rico, decide cambiar radicalmente su vida. Hoy lo conocemos como San Francisco de Asís, quien cuando se refiere al mundo habla del hermano Sol y hermana Luna, del hermano lobo, agua, fuego y árboles y también, de la gente como hermanos y hermanas como un acto amoroso. Un tiempo después también en Italia, se oye la resonante voz del astuto Maquiavelo, advirtiéndonos que "es mucho más seguro ser temido que amado". La ruta que escogimos no fue la de San Francisco, sino la de Maquiavelo e, inspirados en ella, hemos construido nuestros conceptos sociales, políticos y económicos.

Otra manifestación de las bifurcaciones es la de Giordano Bruno, quien arde en la hoguera víctima de su panteísmo, puesto que cree que la tierra tiene vida y alma. Todas las cosas para él son manifestaciones de la vida. Todo es la majestuosa vida. Tres décadas más tarde, René Descartes advierte que a través de su ventana racional lo que ve son sombreros y abrigos que cubren a máquinas automáticas. Aquí tampoco escogimos la ruta de Giordano Bruno sino que optamos por seguir la de Descartes y de esa manera hemos sido testigos del triunfo del mecanicismo, el reduccionismo, el industrialismo y el crecimiento económico y su cosmología dominadora.

Por cosmología se comprende la visión de mundo o cosmovisión que está en la base de las ideas, de las prácticas, de los proyectos y de las utopías de una sociedad. Cada cultura tiene su cosmología. De acuerdo a esta se procura aclarar 
el origen, la evolución y el propósito del universo, e identificar nuestro lugar y relación con el conjunto de los seres o entes que lo constituyen. Se puede considerar la existencia de dos grandes cosmologías: una de dominación y otra cosmología emergente o de transformación.

La cosmología de dominación se caracteriza por ser mecanicista, atomística, determinista, simplificadora, reduccionista. La manera con la cual se relaciona con la vida en la Tierra ha sido tan agresiva que para Wilson (2006). "la especie humana es la primera especie en la historia de la vida en la Tierra que se ha hecho una fuerza geofísica destructiva" (p.38).

Los seres humanos nos hemos organizado, o nos han organizado de tal forma que podamos llevar a cabo una acción implacable contra la tierra, en los suelos, en el aire, en los océanos y en todas partes, y significa que la amenaza a la vida en la Tierra ya no es un meteoro rasante como sucedió antiguamente, que en 15 grandes devastaciones, eliminó gran parte de la vida, sino que ahora la perversa amenaza de autodestrucción es el ser humano (Hathaway, 2014) que antes de reducir, amplía las desigualdades sociales.

Diversas investigaciones han llegado a la conclusión de que, en 2015, el $1 \%$ de la población mundial concentraba en sus manos casi la mitad de la riqueza poseída por el conjunto de las familias del planeta. Asimismo, las 62 personas más ricas del mundo poseen por sí solas tantos bienes como lo que tiene la mitad más pobre de la humanidad.

En el Informe Mundial sobre Ciencias Sociales 2016 se llega concluir que la desigualdad puede poner en peligro la sostenibilidad de las economías, sociedades y comunidades. El Informe también sostiene que la creación de sociedades más equitativas exige un mejor conocimiento de la desigualdad en general, así como de los vínculos existentes entre la desigualdad económica y otras formas de desigualdad, por ejemplo las que se dan en materia de género o en los ámbitos de la educación y la salud (UNESCO, 2016). 
Se enfrenta entonces de manera sistemática un sistema que crea una doble injusticia: una social y otra ecológica. La social conducida por el abismo cada vez mayor entre los pocos ricos y los muchos pobres; la ecológica a partir de la superexplotación de ecosistemas enteros con la enorme desaparición de especies vivas y la desestabilización del equilibrio de la Tierra Patria.

En el devenir de la cosmología de dominación se configura la cosmología de transformación que es emergente, incierta, caótica y potencialmente esperanzadora. Esta sitúa la complejidad de la realidad dentro de un inmenso proceso de evolución no lineal, que se inauguró a partir del big bang. El universo, según esta comprensión, está continuamente expandiéndose, autoorganizándose y autocreándose en una dinámica regeneradora que se reinventa continuamente. Su estado natural es la evolución y no la estabilidad, la transformación, no la inmutabilidad y la permanencia fluyendo, es decir, la creciente complejidad y sus incertidumbres. En este universo todo es relación en redes y nada existe fuera de esta relación, todo está interconectado con todo en un mundo de sistemas. A raíz de esto, todos los seres son interdependientes y colaboran entre sí para co-evolucionar y garantizar la vigencia de todos los factores del equilibrio caótico, cósmico y de la propia Tierra, que se estabiliza a partir del no-equilibrio.

Como la cosmología de dominación globalizada sigue siendo hegemonizadora y decadente, en este momento la cosmología de transformación puede ser generadora y transformadora para cambiar de vía. Esta cosmología de transformación se caracteriza por el cuidado y la solidaridad religadora para potenciar la generación de nuevas posibilidades de vida, en lugar de la dominación; el reconocimiento del valor intrínseco de cada ser en lugar de su instrumentalización y utilización codiciosa y el respeto hacia todas las formas de vida, dignidad y derechos de naturaleza en lugar de la falta de respeto y su explotación ilimitada.

La fuerza de esta cosmología reside en el hecho de estar más acorde con las necesidades humanas reales y con la lógica misma del universo para hacer 
posible la creación de las bases para una política de civilización en el cuidado, la cooperación, la solidaridad con los más débiles, la compasión, el respeto, el amor, la alegría de vivir en dignidad; significaría el gran viraje esperanzador que tanto necesitamos si decidimos aún vivir sobre este planeta. En las circunstancias actuales se necesita actuar en medio de ambivalencias al considerar que junto con todos estos valores positivos nos acompañan también sus contradicciones, porque nuestra condición humana es la de ser sapientes y dementes, portadores de amabilidad y también de enemistad.

\section{Reinventar el desarrollo para generar el buen vivir}

El concepto desarrollo ha sido el instrumento mediante el cual se crea un vasto aparato institucional en el que el discurso se convierte en una fuerza epistemológica para transformar la realidad social, económica, cultural y política de las sociedades, operando mediante la profesionalización de los problemas de desarrollo, incluyendo el conocimiento especializado de amplias áreas del saber.

Se identifica retóricamente el inicio de la época del desarrollo con el discurso del presidente Truman (1949) luego de la Segunda Guerra Mundial, cuando anunció al mundo la nueva doctrina del trato justo para los que desde entonces fueron vistos como áreas subdesarrolladas que deberían seguir los pasos de los países desarrollados para alcanzar el bienestar.

Bajo la cosmovisión de dominación, el campo de análisis del desarrollo se configura durante la posguerra. Es importante considerar que al hablar de desarrollo aludimos a un resultado a alcanzar, mediado por una serie de valores. Esto significaría que en sociedades como las latinoamericanas, caracterizadas por profundas desigualdades estructurales, lo que se está desarrollando es la desigualdad, la pobreza, la injusticia, el deterioro ambiental. Es decir, desarrollamos el subdesarrollo, o por el contrario, se opta por una perspectiva emancipadora con una cosmovisión transformadora, que implica desordenar la realidad en la que vivimos para reinventar y construir otra. Esta es la pregunta que ha atravesado toda la época de violencia que ha experimentado la humanidad. 
En el campo del desarrollo, la teoría ha conocido diversas concepciones que según Larrea (2010) ninguna cuestiona el capitalismo, sino que se promueve el desarrollo humano sustentable en la sociedad capitalista, siendo que capitalismo y sustentabilidad son términos opuestos, pues en las circunstancias es imposible que la sociedad capitalista sea sustentable, porque optar por la sustentabilidad supone afectar las bases del modelo de acumulación fundamentadas en el crecimiento económico y la inequidad, para el que la naturaleza es solamente un recurso más, como se expresa en los planes de desarrollo a partir de la Economía Verde.

El concepto de desarrollo como crecimiento económico ha entrado en una profunda crisis, no solamente por la perspectiva colonialista de la cosmovisión dominadora desde donde se construyó, sino además por los pobres resultados que ha generado en el mundo entero en beneficio de toda la humanidad y sistemas vivos. Las innumerables recetas para alcanzar el supuesto desarrollo, concebido desde una perspectiva de progreso y modelo a seguir de manera homogenizadora imperial-capitalístico, ha llevado a una crisis global de múltiples dimensiones, que demuestra la imposibilidad de mantener la ruta extractivista y devastadora para los países del sur, las desiguales relaciones de poder y comercio entre el Norte y el Sur y los ilimitados patrones actuales de consumo, que sin duda tienen al planeta entero ante la amenaza de un colapso, al no poder asegurar su capacidad de regeneración para preservar el futuro de la vida si no se actúa con celeridad. Es imprescindible entonces, impulsar nuevos modos de producir, consumir y organizar la vida.

Se trata de reorganizar las relaciones de convivencialidad; el objetivo ya no es el desarrollo desde esa perspectiva unilineal de la historia, sino la construcción de una sociedad en una dinámica de amplias relaciones entre los seres humanos, la naturaleza, la vida comunitaria, los ancestros, el pasado y el futuro. Es la construcción de la sociedad del Buen Vivir en el que la economía debe estar al servicio de la vida y no la vida en función de la economía. La transformación hacia la economía social como economía plural, es la transformación hacia 
un pensamiento económico que ha de ser de manera inter y transdisciplinar para que posibilite recuperar el sentido de la oikonomia como el arte de vivir y vivir bien, válida para todos los ciudadanos, satisfaciendo las necesidades y poniendo límites al consumo que pone en peligro la sostenibilidad planetaria.

En esta perspectiva desde la crítica descolonizadora a la economía se comprenden un conjunto de investigaciones que vienen configurando una perspectiva de abordar la economía más allá de los enfoques desarrollistas a partir de la crítica a todo tipo de dominación, que en la perspectiva de una epistemología del sur de los países subalternados proponen una nueva educación para una nueva convivencialidad que se desprenda críticamente de las epistemologías colonizadoras, haciendo visible la interculturalidad y diversidad. No se trata de una cruzada contra la educación y cultura occidental, sino más bien de una ampliación del campo de visibilización; no es la disyunción sino la conjunción epistémica, integrativa entretejiendo caminos de estar, ser, pensar, mirar, escuchar, sentir, vivir con sentido u horizonte descolonial y el enraizamiento con la naturaleza en la construcción del buen vivir (Acosta, 2013).

Hoy precisamos abordar los problemas en sus interdependencias y complejidades sistémicas siendo necesario optar por un cambio profundo en la manera de conocer, pensar, aprender, sentir, ver, comunicar, investigar, puesto que también hemos aprendido incapacidades por las disciplinas que nos enseñaron a separar, desunir. Se trata de un aprendizaje como proceso indeterminado, no lineal, autoorganizador, asociativo, activador de capacidades para reconocer y reflexionar las temporalidades y emergencia de saberes y sentires vinculados al mundo de las trayectorias humanizadoras.

La configuración de saber pensar hoy, significa indisociablemente saber pensar el pensamiento propio, de tal manera que tenemos necesidad de saber ver el mundo, de pensar pensándo-nos, de conocer, conociendo-nos, de investigar investigando-nos en continua armonía con el mundo de la emocionalidad. 
Lo fundamental es considerar la autonomía del ser humano que piensa, aprende, conoce y siente en todo acto de conocimiento. De tal manera que pueda autoanalizarse, ejerciendo una reflexividad circular, que permita las descripciones y la comprensión del propio conocer-vivir-sentir. Solo así construimos este mundo, construyendo conjuntamente el contexto, el espacio, el territorio, la sociedad, la familia, la universidad, es decir haciendo apuestas, siempre en una construcción del tiempo, no como el tiempo calendario, el tiempo reloj sino el tiempo de las temporalidades de cada persona, en el forjar su propia vida. Es el tiempo de la construcción social que se hace con los próximos y con los lejanos en momentos cargados de incertidumbres, metamorfosis y profundos cambios.

La fase actual de la sociedad denominada economía del conocimiento, requiere dar cuenta del conocimiento de la economía, ya que no es solo la economía lo que está en crisis; la enseñanza de la economía también enfrenta una crisis con implicaciones que trascienden las fronteras de la universidad. Uno de los problemas que enfrenta la enseñanza de la economía es la reducción a un solo modelo y paradigma de la economía, como si fuera un cuerpo de conocimiento unificado, cuando en la sociedad históricamente se mueve una pluralidad de concepciones y prácticas en la economía.

Enriquecer los aprendizajes y las investigaciones de manera incluyente implica un pluralismo de teorías, métodos, estrategias, sentidos, cosmovisiones que permitan a todo profesional ampliar los enfoques del pensamiento económico, social, político y cultural vigentes en los planes de estudio. En esta tensión, la incorporación de saberes como la epistemología, filosofía y sociología de la economía en los currículas universitarios así como la necesidad de revitalizar la economía política en función de las políticas públicas, favorecen la contextualización y reflexión sobre la enseñanza de la disciplina económica y sus métodos. Un aspecto a reformar es el aprendizaje del pluralismo metodológico de la economía, pues al lado de las matemáticas y estadísticas, que son las disciplinas recurrentes a una formación de métodos cuantitativos, se requiere in- 
corporar métodos cualitativos propios de las ciencias sociales y humanidades para comprender la complejidad humana, sus instituciones, la diversidad cultural y su convivencialidad con los ambientes ecológicos en que se interactúa.

\section{Los modos de producir conocimiento}

En la cultura occidental han sido dos los grandes caminos que han guiado al pensamiento universal para dar cuenta del conocimiento. Desde el siglo XVII el pensamiento triunfante de Descartes afirmó que "cuando se tiene un problema muy complicado hay que dividir sus dificultades en pequeñas partes $y$ cuando las he resuelto todas, he resuelto el todo". Así se organizaron las ciencias, la educación y los planes de estudio. Una invitación a un pensamiento de ida y vuelta fue el de Pascal, quien decía "No puedo entender el todo si no conozco las partes y no puedo entender las partes si no conozco el todo". Este pensamiento que no fue escuchado, ni siquiera comprendido, está en la actualidad en plena expansión, pues el abordaje transdisciplinar, religador, emergente, dinámico, no-lineal es el que posibilita el tránsito de un sujeto, ser aislado, a un sujeto que intenta ver lo que une unas cosas con otras, es decir mirar no solo la presencia de las partes en el todo, sino también la presencia del todo en las partes. Relacionar lo uno con lo diverso, es precisamente el gran desafío al que se enfrentan tanto el conocimiento como la educación en todos los procesos.

Un nuevo contexto mundial para el aprendizaje y la investigación social ha emergido como respuesta a la crisis del modelo tradicional, y está configurando un nuevo horizonte para la formación profesional del trabajador social.

El papel predominante y casi único del sistema educativo, para crear conocimiento ha sido movilizado por la dinámica vertiginosa de la tecno-ciencia, las necesidades que se plantean la economía y las nuevas tecnologías de la información. Esto ha movilizado el eje de generación del conocimiento para ser compartida esta misión histórica con otros sistemas como el económico, el político, cultural, ciudadano y hoy el ambiental; requiere de la educación superior, articular y curricularizar los diversos saberes en la formación integral que se declara en los proyectos educativos. 
La universidad está enfrentada a un enorme desafío como nunca antes lo había estado. La posición estratégica en el seno de una sociedad que se debate en una tensión histórica, en medio de emergentes desafíos globales plantea una época de transición en función de la sostenibilidad ambiental; el incremento de las desigualdades sociales recurrentes cuando se aborda la formación profesional (Unesco, 2016); las nuevas bases epistemológicas que fundamentan actualmente la ciencia; la prevalencia de las tecnologías de la información y la expansión de los datos producto de la actividad científica; la emergencia de nuevas ciencias y tecnologías, han dado al traste con los modos de pensar y construir los conocimientos heredados de la ciencia clásica y todo su tránsito por la modernidad y la posmodernidad, configurando una nueva perspectiva cultural y epistémica para la educación superior.

A la caracterización del conocimiento como una creación o construcción se ha puesto otra que ve al conocimiento como producción, y es el lenguaje de las ciencias económicas, el que caracteriza privilegio epistemológico, frente al acto mismo del sujeto constructor. Se han configurado los denominados modos de producción del conocimiento a partir de las hélices de diversos sistemas que interactúan como modelos de conocimiento o modelos de innovación (Carayannis \& Campbell, 2010) aparentando ser un proceso democrático, dialógico $\mathrm{y}$ transdisciplinar.

\section{5. Ética y bioética para la comprensión y el sentipensar}

Históricamente se reconoce que ninguna sociedad vive sin una ética. Como seres sociales necesitamos elaborar ciertos consensos, cohibir ciertas acciones y crear proyectos colectivos que dan sentido y rumbo a la historia. Hoy, debido a la globalización se constata el encuentro de muchos proyectos éticos, no todos compatibles entre sí. De cara a la nueva era de la humanidad, ahora mundializada, se siente la urgencia de una base ética mínima que pueda conseguir la aceptación de todos y hacer así viable la convivencia entre los pueblos.

Hoy vivimos una grave crisis mundial de valores. A la inmensa mayoría de 
la humanidad le resulta difícil saber lo que es correcto y lo que no lo es. Ese oscurecimiento del horizonte ético redunda en una enorme inseguridad en la vida y en una permanente tensión en las relaciones sociales, que tienden a organizarse más alrededor de intereses particulares que en torno al derecho y la justicia. Este hecho se agrava aún más por causa de la propia lógica dominante de la economía y del mercado, que se rige por la competencia -la cual crea oposiciones y exclusiones- y no por la cooperación -que armoniza e incluye(Hathaway, 2014).

Por fuerza de la crisis múltiple que experimenta el sistema antropoceno, hoy podemos considerar que estamos en una etapa en transición, lo que es una buena oportunidad para reinventar y activar la creatividad para cuidar e innovar. La crisis ecológica y social ha llevado a muchos visionarios a proponer una transición ecológica y cultural profunda, que se oriente a sistemas de organización socio-naturales muy diferentes a los actuales, que trasciendan los modelos del sistema capitalista.

Los discursos en transición, los identificamos como emergentes, convirtiendo a la emergencia en una categoría epistemológica indispensable para comprender y actuar en el mundo de hoy, y aprender a vivir en convivencialidad, lo que quiere decir que, hay que tener presente una ética devenida en bioética.

La bioética más allá de una disciplina, implica una comprensión, una preocupación y una ocupación por la vida en general, no solamente la vida humana, sino también la vida en general sobre el planeta. Tanto la vida conocida tal como ha sido, como la vida que hacemos y la vida como podría ser, es decir, la vida por conocer.

El tratamiento y comprensión de la vida es un problema de frontera, es un campo abierto, cuya comprensión y explicación no son exclusivos de la medicina, de la biología, del derecho, de la religión, de la filosofía, de la ecología, de la tecnología, de los gimnasios. 
De esta manera los temas de la bioética requieren ser comprendidos y tratados en su complejidad, precisamente por ocuparse del estudio de los procesos, fenómenos, comportamientos y sistemas que exhiben, contienen o presentan vida que son los más complejos. La bioética, al ocuparse del fenómeno de la vida, bien para entenderlo, como fenómeno que evoluciona requiere ser tratado de manera inter y transdisciplinar.

En el abordaje bioético corresponde una reelaboración del concepto de ética, por lo que vale la pena replantearse la moral, lo que puede ser entendido como mucho más próximo a la vida social ciudadana, es decir, a la biopolítica, a los derechos humanos, a la justicia social, al derecho a la vida digna, a la antropoética. Es de esperar que una reforma moral pueda volver a colocarla en el centro de la subjetividad humana y social como una cultura para la vida. Esta reforma moral necesita de la integración en la conciencia y la personalidad de cada uno y de un principio de auto-examen permanente, porque sin saberlo nosotros mismos nos mentimos, nos engañamos sin cesar (Morin, 2011).

De igual manera se requiere desarrollar la aptitud para entender a los demás, pues la ética de la vida en sociedad, es la del ciudadano que debe asumir sus deberes y derechos con y en la colectividad. La dimensión de la Bioética, es hoy la Ética del género humano para la metamorfosis necesaria de la humanidad, necesitándose conjugar varias reformas: políticas, económicas, sociales, educativas, de pensamiento, de la vida y la Moral, que en definitiva dependen de cada uno de nosotros.

Una de las necesidades actuales de la sociedad es la de la ética, entendida como los valores que tiene el ser humano a pesar de las diferencias. La ética compleja es entonces la comprensión, el entendimiento, no solo con los más próximos, con la familia, los amigos, los colegas, sino también con los otros; es también la relación entre el saber y el actuar, conexión que debemos buscar de manera cotidiana y en todos los ámbitos y dimensiones de la vida individual y social. Es una manera de orientar tanto el pensamiento como la acción. 
Pensamiento complejo significa una nueva comprensión del futuro humano, que significa desarrollo conjunto de las autonomías individuales, de las buenas voluntades, de las participaciones comunitarias y del sentido de pertenencia a la especie humana, pues dentro de esta tríada compleja emerge la conciencia que es la base para enseñar la ética compleja, que es una antropoética en la medida que se propone asumir la condición humana individuo-sociedad-especie, en la complejidad de nuestro ser, lograr la humanidad en nuestra conciencia personal de asumir el destino humano desarrollando la ética de la solidaridad, de la comprensión, de la compasión, de la fraternidad, de la solidaridad, del perdón, del género humano.

Para el pensamiento complejo la ética compleja es una ética política que supone primordialmente la restauración del sujeto responsable, lo que depende de diversas exigencias como la autocrítica personal, la conciencia de la complejidad humana así como de los diversos caminos itinerantes de todas las personas, y una moral que logre la comprensión y tolerancia de la multipersonalidad de cada individuo, complejo para no reducir al ser humano a uno de sus actos ni alejarlo de la humanidad. De esta manera, en la ética compleja, la comprensión debe preceder y proceder al juicio, comportando una auto-ética como conciencia de la responsabilidad personal, social y de la humanidad. La restauración del sujeto responsable tiene como exigencia la auto-reflexión, la conciencia de la responsabilidad personal, así como asumir autónomamente la ética, es decir, la auto-ética.

Para el pensamiento complejo la responsabilidad requiere situarse en términos complejos, en el que cada uno debe reconocer-se responsable por sus palabras, por sus escritos, por sus actos., pero al mismo tiempo, opera una ecología de la acción en la que ninguno es responsable por el modo como sus palabras son entendidas, como sus escritos son comprendidos y como sus actos son interpretados. 
La ética compleja cuenta así con unas ideas guía

a. La ética del religar. La noción de religar comprende todo aquello que se es capaz de comunicar, solidarizar, fraternizar. Asociar.

b. La ética del diálogo, del debate. El debate es inherente a las instituciones democráticas, exigiendo la primacía de la argumentación.

c. La ética de la comprensión. La comprensión permite conocer al sujeto, por eso es complementaria a la explicación

d. La ética de la magnanimidad, la clemencia, la generosidad, la nobleza.

e. La ética de buenas voluntades. La humanidad hoy tiene que volver a apelar a las buenas voluntades de todos, a solicitar que exista asociación entre sí para salvarla de un desastre. En la educación es necesario re-educar a los educadores para que estos puedan orientar a los estudiantes en lo que sería una educación en convivencia apelando a las buenas voluntades, que no será suficiente mientras no se tenga la voluntad y la capacidad de comprender a nuestros semejantes. Esta es una ética por la supervivencia de la especie humana en el planeta.

f.- La ética de la resistencia. La resistencia constituye tanto la primera como la última de las éticas para este tiempo de tránsitos y crisis, especialmente frente a la barbarie que se amplía cada vez más al interior de la actual sociedad.

\section{Conclusiones}

La complejidad del religaje consiste en tratar de comprender la emergencia, lo emergente, lo que surge, lo que está naciendo, lo que genera la crisis y sus nuevas posibilidades. Religar los saberes ya dados, existentes, es un primer nivel de una relación simple, que a veces se conjugan como adiciones como la multidisciplinariedad.

Si lo emergente está excluido y no lo vemos, el acto creativo consiste en develar, revelar el tercero excluido, lo entramado, lo que está escondido y por esto la actitud de reinventar es activadora de la creación de nuevas posibilidades. 
Lo emergente tiene la apariencia de novedad espontánea y aunque no puede decirse que surge de la nada, tiene una cualidad de discontinuidad.

En los emergentes hay una cooperación entre cosas, sujetos, acciones, energías de clases dispares. El emergente se da por un proceso de asociación.

En este emergente, el Iinter-trans, es el de todos y no es de nadie, por eso posibilita que las diferencias accedan a él; es el complementario, lo que posibilita conectar es lo emergente, lo que está dándose, no lo dado.

El religar es en sí mismo una complejidad que anima al pensamiento para comprender Lo Religado como lo pasivo, lo dado, lo elaborado, Lo Religante como lo participante y la Religación como lo activante del pensamiento y las acciones del saber pensar.

Religar saberes implica resignificar para reinventar. Significar es un acto de representar lo dado, resignificar es un acto de desobediencia epistemológica, pues se trata de desconectar para conectar de nuevo, ahora enriquecido con lo emergente.

Se necesita una epistemología de segundo orden para pasar del saber al comprender comprendiendo-nos, relacionando-nos, para dar cuenta de sí mismo en la apertura al otro y la otra de una realidad en la que estamos inmersos.

La noción de convivencialidad ecosistémica es religadora. Una epistemología que religue, que ayude a conectar a articular y poner en movimiento las acciones solidarias entre los humanos, y de este con toda forma de vida considerando a la madre tierra como ser vivo, esta es la apuesta del sentipensar como una manifestación de construcción de nuevos territorios de paz.

\section{Referencias Bibliográficas}

Acosta., A. (2013). El Buen Vivir. SumakKawsay, una oportunidad para imaginar otros mundos, Barcelona: Ed. Icaria. 
Carayannis, E. \& Campbell, D. (2010). Triple Helix, Quadruple Helix and Quintuple Helix and how do knowledge, innovation and the environment relate to each other? A proposed framework for a trans-disciplinary analysis of sustainable development and social ecology. International Journal of Social Ecology and Sustainable Development. 1(1), 41-69.

CICS/UNESCO (2016). Informe mundial sobre las ciencias sociales. UNESCO. París.

Guattari, F. y Rolnik, S. (2005). Micropolítica. Cartografías del deseo. Madrid: Traficantes de sueño.

Fontalvo., R. (2009). Complejidad y Ciencias Sociales Humanas. Barranquilla: EdicionesUniversidad Simón Bolívar.

Gudynas., E. (2012). Debates sobre el desarrollo y sus alternativas en América latina. Una breve guía heterodoxa. En más allá del desarrollo. Cali: Ediciones AbyaYala.

Hathaway., M \&Boff., L. (2014) El Tao de la Liberación. Una ecología de la transformación, Madrid: Editorial TROTTA.

Larrea., A. (2010). La disputa de sentidos por el buen vivir como proceso contra hegemónico. En Los nuevos retos de América Latina Socialismo y SumakKawsay. Quito: Ed. SENPLANDES.

Max-Neef., M \& Smith., P.(2011). La economía desenmascarada. Del poder y la codicia a la compasión y el bien común. Barcelona: Icaria.

Morín., E. (2011). La vía para el futuro de la humanidad. Barcelona:Paidós.

Wilson, E. (2006). La creación: salvemos la vida en la Tierra. España: Romanyà Valls S.A. 